\title{
Numerical Simulations Research for Applications in Binary Distillation Using Visual Basic for Application (VBA) Programming and Ponchon-Savarit Method
}

\author{
Allan Paolo L. Almajose, and Lola Domnina B. Pestaño
}

\begin{abstract}
Microsoft (MS) Excel, a spreadsheet application by Microsoft Corporation that features a macro programming language called Visual Basic for Applications (VBA) is employed as an engine for the development of numerical simulation programs for distillation. Multiple numerical techniques, MS Excel's built-in tags and codes and the circular-reference solution technique are utilized to achieve a converged and accurate value. The research attempts to formulate numerical simulation programs for the Ponchon-Savarit methods for binary non-ideal distillation using automated calculations for determining the number of plates in a plate distillation column with non-constant molar overflow. Generating distillation applications programs in MS Excel spreadsheet is possible by implementing numerical methods as well as automating tasks such as data organization in VBA, equipped with high coding effort and knowledge in numerical solution algorithms. MS Excel's excellent graphical representations provide the user a reinforced intuition in the calculations. The results are found to be superior in accuracy and calculation speed as compared to the analytical and graphical methods of solution noting the 15-digit floating point accuracy limitation as defined by IEEE-754 standards.
\end{abstract}

Keywords - distillation, microsoft excel, numerical simulation, ponchon-savarit, spreadsheet.

\section{INTRODUCTION}

In the Philippines, applications on distillation requires the students to perform calculations involving design to simulate distillation processes for the separation of any binary mixture. At present, the simplistic and graphical method provided by McCabe and Thiele [1] is virtually discussed in most unit operations textbooks by McCabe, Geankoplis and Foust [1]-[3], and is still being taught in the classroom. This method is extremely limited and inconvenient for non-constant molal overflows, and involving enthalpy balances in the calculations result to the difficulty of teaching it without hopelessly being bogged down in the analysis. The long and complex calculations cannot be completed in one single session as a classroom assessment.

In the information age, there have been more visual - spatial learners in schools. Visual - spatial learners are individuals

Manuscript received April 27, 2016

A. L. Almajose was a student of the University of Santo Tomas. He is now an independent numerical simulations specialist in Manila, Philippines

L. B. Pestaño is with the Department of Chemical Engineering, Faculty of Engineering, University of Santo Tomas, España, Manila, Philippines who think in pictures rather than in words. They have a different brain organization than auditory - sequential learners. They learn better visually - demonstration over oral lectures according to Silverman [4]. There is a growing awareness of developing students' computer skills in engineering courses. Simulations-assisted learning is necessary to enhance the theoretical concepts and to guide the engineering students with a major advantage in their future careers. In the field of process systems engineering (PSE) the mathematical programming computer methods have led to successful results for real industrial applications according to Garcia and Caballero [5].

Microsoft Excel (MS Excel) is the most commonly used spreadsheet application available in almost all computers. The application has been used in modern teaching and solving various mathematical problems in all branches of science and engineering. Numerical methods can be translated into MS Excel tags and codes that could create a calculating tool for engineering applications. MS Excel's interface allows users to manipulate and design the spreadsheet to generate useful simulations as stated by El-Gebeily and Yushau [6].

This numerical simulations research aims to formulate an MS Excel program for the Ponchon-Savarit distillation method that will associate and use various numerical methods and translate MS Excel functions into replicating various numerical technique mechanisms.

\section{A. Significance}

The developed MS Excel programs will provide a straightforward solution to distillation processes using the Ponchon-Savarit method for non-ideal binary separation. The programs developed are primarily designed for the process analysis instruction of distillation processes. A unique distillation process problem may be assigned to each and every student in a distillation unit operation class by varying the amount of feed, type of feeding and the desired computational parameters, such as operating pressures, temperatures, binary phase equilibrium $(y-x)$ data, enthalpy-concentration $(H-x)$ data, among others. Questions may be handed as a take-home exam and students will work independently with their respective problem set outside the classroom where they can be able to think, study, and analyze the calculation processes, thereby understanding the distillation unit operation. Confirmation of the students' unique solution and final answers shall no longer be a burden to the instructor since the tedious trial and error method is eliminated by using the 
simulator.

\section{METHODS}

\section{A. Numerical Simulations using MS Excel}

MS Excel tags and codes are used in conjunction with various numerical techniques in organizing numerical solutions for the simulation of the distillation process given certain scopes and limitations. MS Excel's iterative solution coding and circular-referencing are used in generating the simulators in order to save space and computer memory in exchange for eliminating the initial iterations. AutoShapes included in MS Excel's mainframe are used to draw the distillation diagrams, most importantly the stage and plate-by-plate separation diagrams, reinforcing the intuition of the user as stated by El-Gebeily and Yushau [6]. Fig. 1 shows the interface of the binary rectification simulator.

\section{B. Programming}

The simulator program initially requires $H-x$ and $y-x$ data, generated either from either experiments or from other phase equilibrium generators such as ChemSep [7]. The $H-x$ and the $y$ - $x$ data are then regressed to high-order polynomials using MS Excel's =linest() function. The developed equations followed the generic form (1) and (2) for vapor-phase and liquid-phase enthalpies and (3) for the phase equilibrium fractions. The user may then choose the best fit for each data set by graphical comparison of the data points with the generated curve.

$$
H_{V}\left(x_{n}\right)=A_{H}+B_{H} x_{n}+C_{H} x_{n}^{2}+D_{H} x_{n}^{3}+\ldots+\Psi_{H} x_{n}{ }^{n} .
$$

$$
\begin{gathered}
h_{L}\left(x_{n}\right)=A_{h}+B_{h} x_{A}+C_{h} x_{A}{ }^{2}+D_{h} x_{A}{ }^{3}+\ldots+\Psi_{h} x_{A}{ }^{n} . \\
x_{n}=A_{x}+B_{x} y_{n}+C_{x} y_{n}{ }^{2}+D_{x} y_{n}{ }^{3}+\ldots+\Psi_{x} y_{n}{ }^{n} .
\end{gathered}
$$

The program then employs mass balances (4) and (5) for flow rates of the simulated fluid flow rates of the feed $\mathrm{F}$, distillate D, and bottoms B and are solved using linear substitution methods.

$$
\begin{gathered}
F=D+B . \\
F x_{F}=D x_{D}+B x_{B} .
\end{gathered}
$$

In coding the spreadsheet, the $H$-x points for $F, D, B$, liquid distillate enthalpy $L_{0}$ and vapor distillate enthalpy $V_{l}$ are determined from the inputted data of the user. Using points $L_{0}$, $F$ and $V_{l}$, the enthalpy points $D^{*}$ and $B^{*}$ are calculated by the spreadsheet as shown in Fig. 2. The determination of the points $D^{*}, F$ and $B^{*}$ are required as they will form a straight line that designate the rectification and stripping zones and are then plotted, as shown in Fig. 3. Further, the enthalpy readings for points $D^{*}$ and $B^{*}$ correspond to the heat load needed to effect successful separation for a distillation process.

Point $D^{*}$ is located at the point $\left(x_{D}, h_{D}+q_{C D}\right)$ in the $H-x$ diagram; thus the numerical value of $h_{D}+q_{C D}$ must be determined. It is calculated by codified form of the inverse-lever arm rule, as formulated by (6) and (7).

$$
\frac{L_{o}}{D}=\frac{\left[h_{D}+q_{C D}\right]-H_{V}\left(x_{D}\right)}{H_{V}\left(x_{D}\right)-h_{L}\left(x_{D}\right)} \text {. }
$$

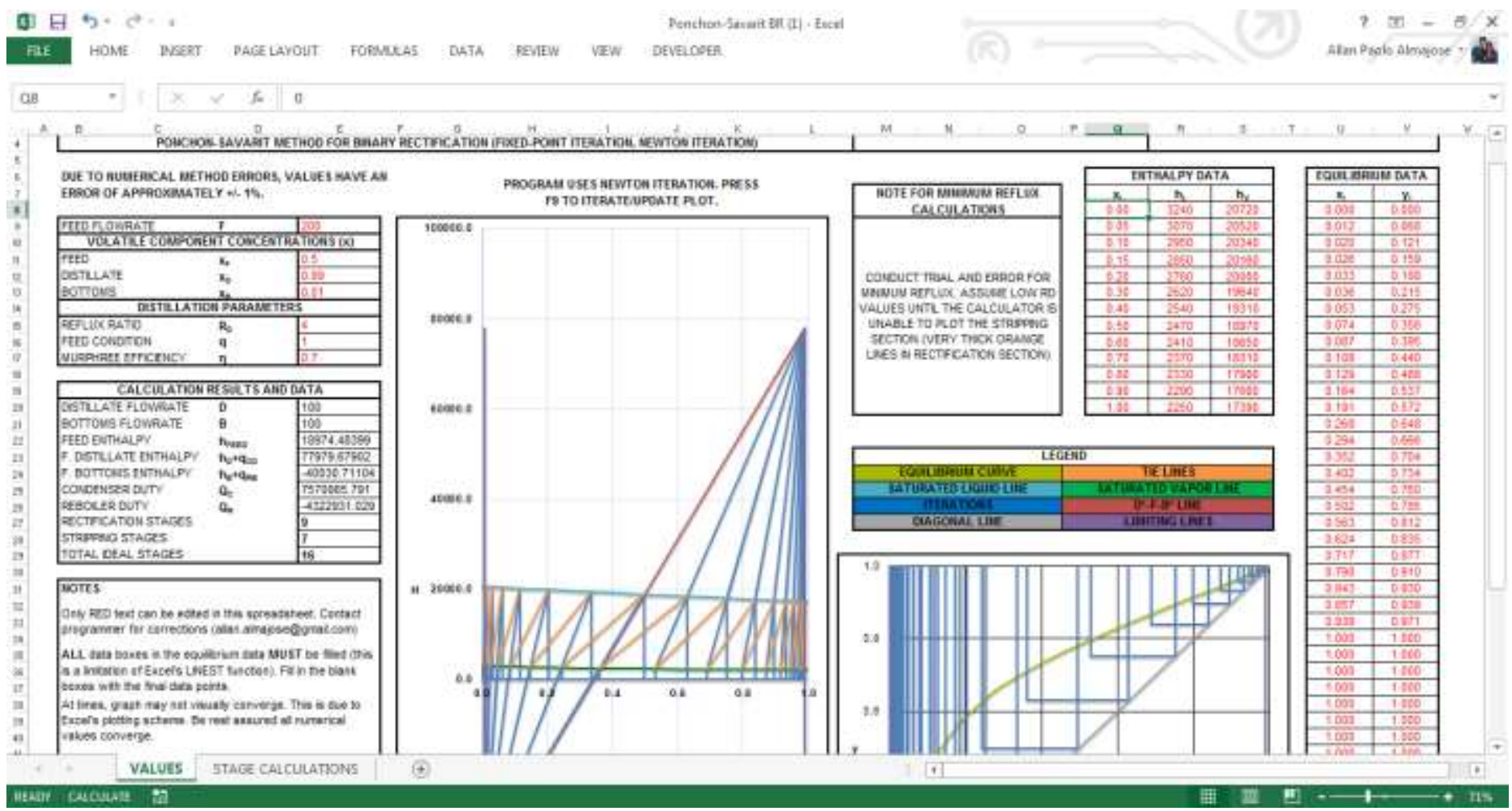

Fig. 1. Interface of the Ponchon-Savarit distillation simulator. Red text denotes editable regions 


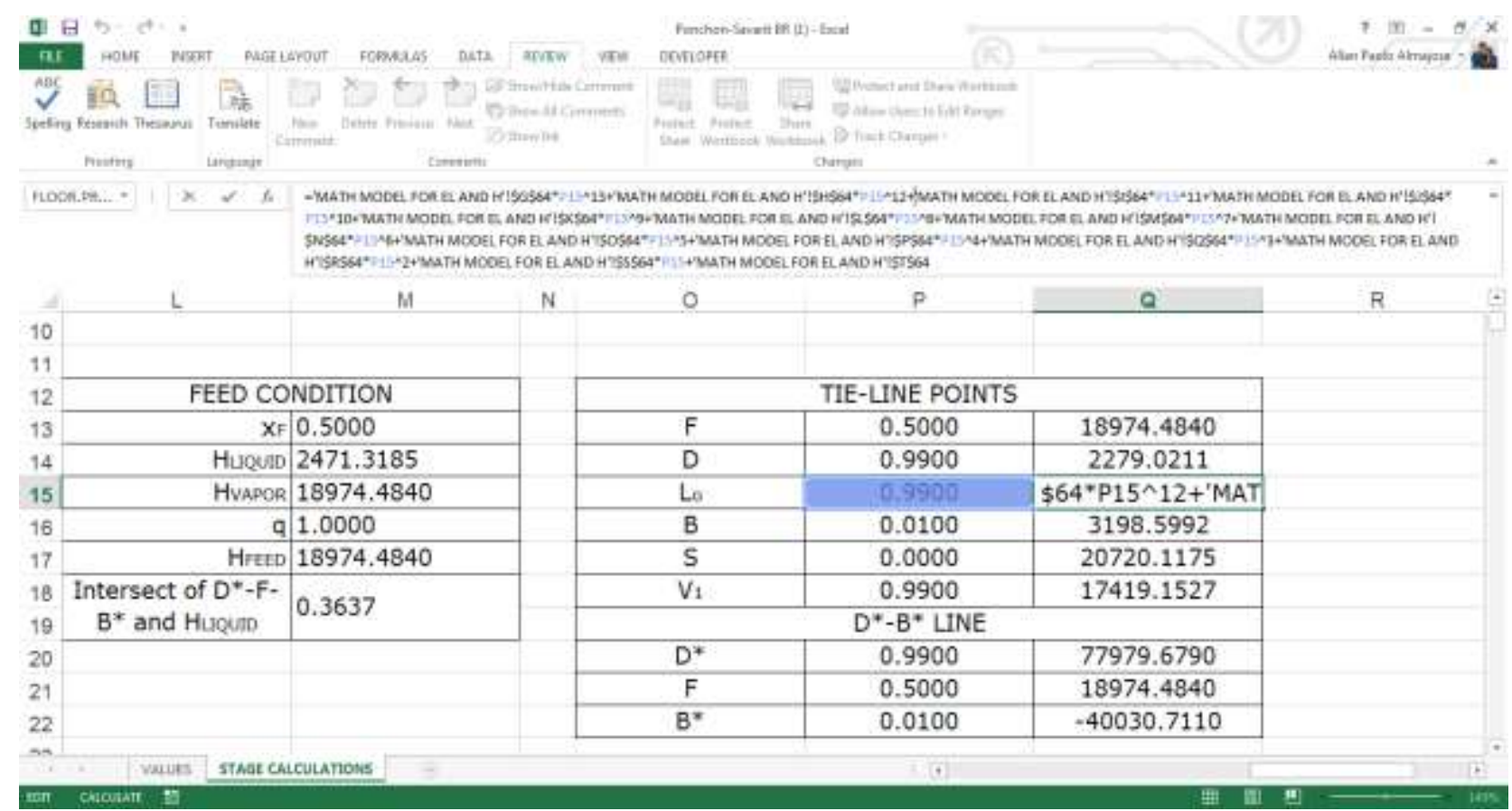

Fig. 2. Evaluation panel of the enthalpy points for the $D^{*}-F-B^{*}$ line from the polynomial equations.

$$
h_{D}+q_{C D}=R_{D} \cdot\left[H_{V}\left(x_{D}\right)-h_{L}\left(x_{D}\right)\right]+H_{V}\left(x_{D}\right)
$$

Point $\mathrm{F}$ is determined from $\left(x_{F}, h_{F}\right)$ taking into consideration the ordinate of the point by (8), which is dependent on the value of $q$, the ratio of the feed flow rate that is vaporized, as described by McCabe, Smith and Harriott [1]. The two-point formula of a line is then used to construct a linear equation to locate point $B^{*}$ with coordinates $\left(x_{B}, h_{B}+q_{R B}\right)$ by using the points generated by $D^{*}$ and $F$ as shown by (9).

$$
\begin{aligned}
h_{F} & =H_{V}\left(x_{F}\right) \cdot q+h_{L}\left(x_{F}\right) \cdot(1-q) . \\
h_{B}+q_{R B} & =\frac{\left[h_{D}+q_{C D}\right]-h_{F}}{x_{D}-x_{F}}\left(x_{B}-x_{F}\right)+h_{F} .
\end{aligned}
$$

The diagonal line equation (10) as described by Seader and Henley is used to establish stage-to-stage equilibrium calculations [8].

$$
x_{n}=y_{n} \text {. }
$$

Simulation of the amount of plates for the distillation process is done by substituting the distillate concentration $x_{D}$ to (1) and (2). Equilibrium stage-to-stage calculations are then achieved by (10) before substitution to (3) for phase equilibrium concentration changes. The resulting concentration change $x_{n+1}$ is reevaluated by (2) to calculate the new liquid enthalpies and plotted, as shown in Figs. 4(a) and 4(b).

The simulator determines the location of the updated concentration value $x_{n+1}$ by conducting a check whether the value $x_{n+1}$ is greater, equal or less than the intersection of the $D^{*}-F-B^{*}$ line and (2). After locating $x_{n+1}$, the new point $\left(x_{n+1}, h_{L, n+1}\right)$ is forced to form a linear equation with $D^{*}$ for the rectification zone or $B^{*}$ for the stripping zone, dependent on the location of $x_{n+1}$. The resulting intersection of this linear equation with (1) found using the Newton-Raphson iteration represents the $n^{\text {th }}$ stage of the distillation process, corresponding to the $n^{\text {th }}$ plate of a distillation tower. Further iterations are conducted until $x_{n}$ reaches a value less than the bottoms concentration, $x_{B}$.

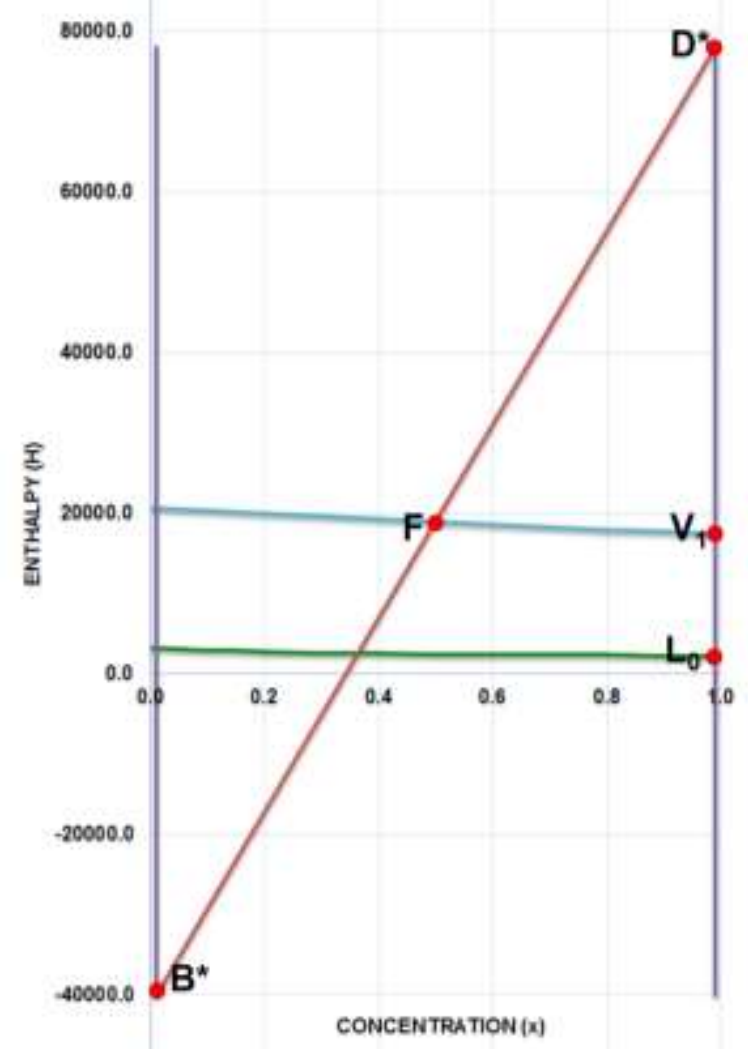

Fig. 3. Construction of the $D^{*}-F-B^{*}$ tie line where $q$ is 1 , separating the rectification and stripping zones as generated by the enthalpy points. 


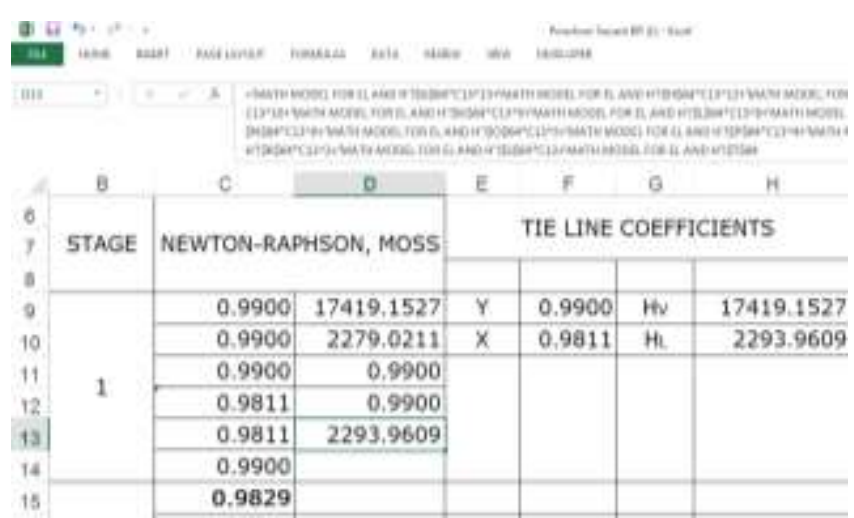

Fig. 4(a). The diagonal line $V_{l}-L_{l}$ denotes the tie line for enthalpies.

For highly non-ideal distillation processes, Murphree efficiencies represented by $\eta$ are calculated in between the equilibrium evaluations. The Murphree-calculated concentration $x_{n+1}, \eta$ evaluated by using (11) is used instead of the phase equilibrium calculation for the next equilibrium stage.

$$
x_{n+1, \eta}=x_{n} \cdot(1-\eta)+x_{n+1} \cdot \eta
$$

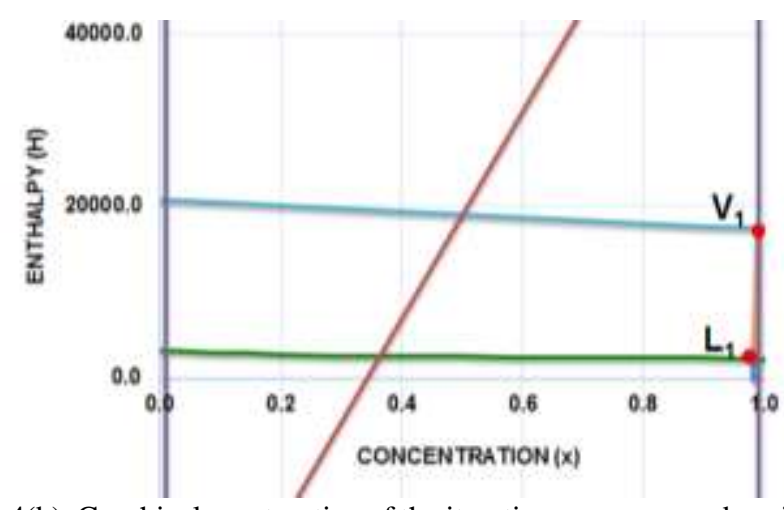

Fig 4(b). Graphical construction of the iteration sequence as described by

\section{A. Simulation set-up}

Two simulators for the Ponchon-Savarit distillation process have been developed, one using simple binary rectification, and the second using open-steam binary rectification processes. Though different in the mathematical formulation of the stripping zone, they have the same method of coding.

As shown in Fig. 5, the user enters the desired distillation process parameters such as the $y-x$ and $H-x$ data, operating pressures, temperatures, feed quality, among other required parameters. To calculate the results, the user will have to update the spreadsheet by pressing F9 key in order for the distillation simulator to begin the iterations, which automatically computes the converged and required number of plates, both for ideal and non-ideal distillation whose solution mechanism is described by Seader and Henley [8]. Heat load for distillation to effect are also calculated, taken at the bottom left panel of the spreadsheet.

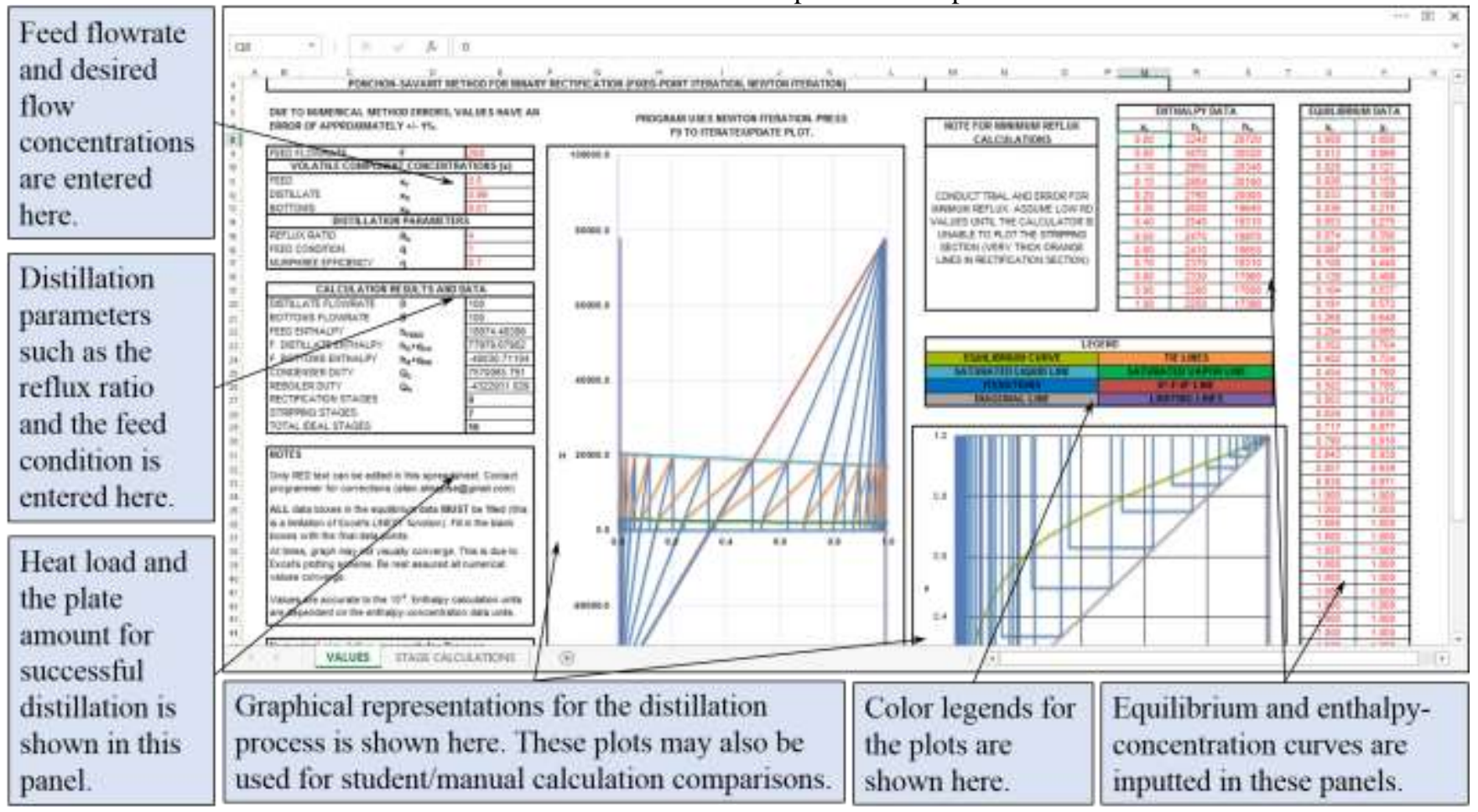

Fig. 5. The simulation setup for a distillation process using the Ponchon-Savarit distillation process simulator program 


\section{III.CONCLUSIONS AND RECOMMENDATIONS}

The MS Excel programming aspect and VBA coding allows the user to implement a wide variety of numerical methods for solving distillation problems and then reporting the results back to the spreadsheet. Further, the simulator's accuracy and speed of convergence are highly reliant on the numerical solution method used. The solution programs work best with the combination of higher-order numerical techniques and the smallest possible step size, keeping in mind the 15-digit floating point limitation of the solution engine by Microsoft Corporation [9], and the possible increase in computation times and coding methods for the usage of higher-order numerical techniques.

Specialized versions of the Ponchon-Savarit simulator will be made available for use by students taking up distillation-related courses. The program will enhance the creativity of the students in the design of distillation towers, to develop their theoretical concepts and guide them with a major advantage in their future careers.

\section{ACKNOWLEDGMENT}

The authors wish to thank the Office of Research and Innovation of the University of Santo Tomas for giving us the permission to represent the university in the RET-2016.

\section{REFERENCES}

[1] McCabe, W., Smith J., and Hariott P., Unit operations of Chemical Engineering, 6th ed., New York, U.S.: McGraw-Hill Chemical Engineering Series, 2001, ch. 21

[2] Geankoplis, C.J., Transport Processes and Separation Process Principles, 4th ed., Upper Saddle River, N.J., US: Prentice-Hall, 2003, ch. 11

[3] Foust, A., Principles of Unit Operations, 2nd ed., New York, U.S.: John Wiley \& Sons, 1980, ch. 4.

[4] Silverman L. "At-Risk Youth and the Creative Process", Gifted Development Center, 2009

[5] Garcia N, Ruben R. and Caballero, J., "Teaching mathematical modeling software for multiobjective optimization in chemical engineering courses". Education for Chemical Engineers, vol. 7, p. 169, 2012. http://dx.doi.org/10.1016/j.ece.2012.07.001

[6] El-Gebeily M. and Yushau B., "Numerical Methods with MS Excel". The Montana Mathematics Enthusiast, vol. 4, no.1, p. 84, 2012.

[7] Kooijman, H., Taylor, R. The ChemSep Book, 2nd ed., Amsterdam, The Netherlands, 2006.

[8] Seader, J.D., Henley, E.J., D.K. Roper., Separation Process Principles, 3rd ed., New York, U.S.: Wiley and Sons, 2013, ch. 7.

[9] Microsoft Corporation (November 2013). Floating-point arithmetic may give inaccurate results in Excel (IEEE-754). Microsoft Corporation. [Online] Available: http://office.microsoft.com/en-001/excel

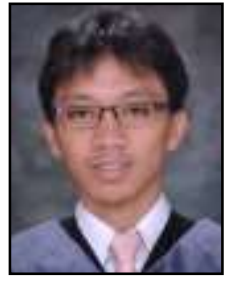

Allan Paolo L. Almajose is an independent researcher on numerical simulations in chemical engineering. A licensed chemical engineer, educator and programmer, he earned units in professional education from the University of the City of Manila (PLM) last November 2016 and is a chemical engineering graduate of the University of Santo Tomas (UST) last March $2014 \mathrm{He}$ currently teaches Science - Technology - Engineering Mathematics-related subjects and is a programming consultant in Montessori de San Juan in Manila, Philippines; a consultant for intermediate to advanced mathematical modeling and numerical simulations in UST Chemical Engineering Department; and a former review specialist for chemical engineering graduates in licensure examinations. Engr. Almajose is currently interested on advanced mathematical simulations and modelling on unit operations and reaction kinetics in chemical engineering.

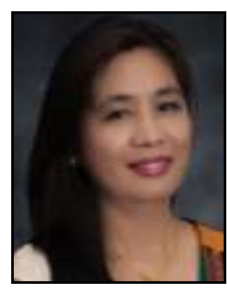

Lola Domnina B. Pestaño is a professor of chemical engineering at the University of Santo Tomas (UST), Manila where she earned her baccalaureate degree in chemical engineering in 1981. She became the chairperson of the UST Chemical Engineering Department in AY 2004-2006 and then in AY 2012-2013. She teaches Transport Processes, Heat and Mass Transfer, Advanced Mathematics, Chemical Reaction Engineering, Particulate Technology and Equipment Design. She received her master's and doctorate degrees in chemical engineering at the University of the Philippines-Diliman (UPD), Quezon City in 1993 and 2014 respectively. Dr. Pestaño looks forward to incorporating unit operation simulations in chemical processing industries. 